\title{
Icariin and Icariside II Reciprocally Stimulate Osteogenesis and Inhibit Adipogenesis of Multipotential Stromal Cells through ERK Signaling
}

\author{
Dawei Zhang $\mathbb{D}^{1,2}$ Ning Zhao, ${ }^{1}$ Chao Wan, ${ }^{1}$ Jikun Du, ${ }^{1}$ Jiantao Lin, ${ }^{2}$ and Hongbo Wang $\mathbb{D D}^{1}$ \\ ${ }^{1}$ Shenzhen Hospital of Integrated Traditional Chinese and Western Medicine, Guangzhou University of Chinese Medicine, \\ Shenzhen 518104, China \\ ${ }^{2}$ Guangdong Key Laboratory for Research and Development of Natural Drugs, The Marine Biomedical Research Institute, \\ Guangdong Medical University, Zhanjiang 524023, China \\ Correspondence should be addressed to Dawei Zhang; dwzhanghk@gmail.com and Hongbo Wang; 412324408@qq.com
}

Received 9 July 2021; Revised 12 November 2021; Accepted 18 November 2021; Published 16 December 2021

Academic Editor: Hamid Tebyanian

Copyright (C) 2021 Dawei Zhang et al. This is an open access article distributed under the Creative Commons Attribution License, which permits unrestricted use, distribution, and reproduction in any medium, provided the original work is properly cited.

\begin{abstract}
Herba Epimedii is a famous Chinese herbal medicine for treating bone diseases. Icariin and icariside II, the main chemical constituents, have attracted great attention from scientists for their potential as antiosteoporosis agents. Our study aimed to evaluate their effects on the lineage commitment of multipotential stromal cells (MSCs). The osteogenesis and adipogenesis of MSCs were assessed by ALP activity, calcium deposition, and adipocyte formation. The expression profiles and levels of osteogenic and adipogenic specific genes were evaluated by cDNA microarray and quantitative real-time PCR. The involvement of extracellular signal-regulated kinase (ERK) signaling was studied by enzyme-linked immunosorbent assay. Icariin and icariside II significantly increased ALP activity and mineralization during osteogenic differentiation of MSCs. Runx2, Col1, and Bmp2 were upregulated in the presence of icariin and icariside II. Meanwhile, they downregulated Pparg, Adipsin, and Cebpb expression during adipogenic differentiation. cDNA microarray revealed 57 differentially expressed genes during lineage commitment of MSCs. In addition, icariin and icariside II enhanced the phosphorylation of ERK, and the above biological effects were blocked by ERK inhibitor U0126. Icariin and icariside II may drive the final lineage commitment of MSCs towards osteogenesis and inhibit adipogenesis through the ERK signaling pathway. Both of them exert multiple osteoprotective effects and deserve more attention for their medicinal and healthcare prospects.
\end{abstract}

\section{Introduction}

The bone is a highly dynamic tissue which undergoes constant remodeling all through life. Normal bone remodeling requires the appropriate balance between bone formation and resorption [1]. Impairment in the bone remodeling process often results in abnormal metabolic bone diseases, especially osteoporosis. Osteoporosis, particularly for postmenopausal women, accelerates the bone resorption process and increases the risk of bone fragility and fractures. Therapeutic options for osteoporosis include antiresorptive agents (bisphosphonates, raloxifene, and estrogen) and anabolic agents (teriparatide and abaloparatide) [2]. However, the pharmaceutics are associated with various side effects and high costs [3].
Herba Epimedii (Yin Yang Huo) has been prescribed for impotence, arthritis, numbness, and weakness of the limbs for centuries in China. According to Chinese Pharmacopoeia, Herba Epimedii is derived from the aerial part of the Epimedium species including $E$. koreanum Nakai, E. brevicornum Maxim, and E. sagittatim (Sieb \& Zucc.) Maxim. As the most frequently used Chinese medicinal herb for osteoporosis and fracture, 85 clinical trials were conducted using traditional Chinese medicine prescriptions containing Herba Epimedii to treat primary and secondary osteoporosis from 2005 to 2016 [4]. Many studies have been performed to investigate the antiosteoporosis effects of Herba Epimedii extracts and chemical constituents, especially icariin, in various animal and cell models. Herba 
Epimedii aqueous extract $(110 \mathrm{mg} / \mathrm{kg} / \mathrm{d})$ protected bone loss in ovariectomized (OVX) rats by improving trabecular microarchitecture and urinary calcium excretion [5]. In addition, total flavonoids of Herba Epimedii $(50$ or $100 \mu \mathrm{g} / \mathrm{g}$ ) were able to suppress urinary calcium excretion as well as loss of bone mass and strength at the distal femur and improve trabecular bone microarchitecture in OVX mice [6]. Administration of icariin $(125 \mathrm{mg} / \mathrm{kg} / \mathrm{d})$ significantly increased biomechanical strength and improved biochemical and histopathological parameters in OVX rats [7]. Chen et al. [8] also reported that icariin $(125,250$, and $500 \mathrm{mg} / \mathrm{kg} /$ d) significantly increased bone mineral density, biomechanical strength, and relieved trabecular microarchitecture deterioration in OVX rats.

Many studies have revealed that flavonoid components of Herba Epimedii exhibited osteoprotective effects by stimulating osteoblast differentiation and suppressing osteoclast differentiation [9-11]. In recent years, there has been an increased amount of interest in the multipotent differentiation capabilities of multipotential stromal cells (MSCs). Flavonoids of Herba Epimedii were reported to stimulate the osteogenesis of human MSCs [12]. Icariin and rat MSCs combined treatment promoted angiogenesis and neurogenesis after cerebral ischemia by reducing brain infarction volume and improving neurologic deficits of motor and somatosensory function [13]. Icariin was able to protect against iron overload induced apoptosis and dysfunction of MSCs [14]. Icariin $(10 \mu \mathrm{M})$ may recover the decreased osteogenic differentiation and bone formation function of MSCs from OVX rats [15]. However, these studies were focused on the protective and osteogenic effects of icariin in MSCs. The reciprocal regulation potential of icariin and its metabolites during osteogenesis and adipogenesis of MSCs remains unclear. Previously, icariin and its metabolites were investigated [16] and reported to regulate the lipid metabolism [17]. Notably, we found that icariin significantly suppressed adipocytic transdifferentiation of osteoblasts [18], which suggests that icariin may have the potential to regulate adipogenesis. Studies have demonstrated that there may exist a reciprocal relationship between osteogenesis and adipogenesis of MSCs [19, 20], and the extracellular signalregulated kinase (ERK) signaling is closely related to this process [21]. Herein, we aimed to investigate how icariin and its main metabolite (icariside II) modulate the lineage commitment of MSCs.

\section{Materials and Methods}

2.1. Cell Culture. MSCs were isolated from 6-week-old Kunming mice as previously described [22, 23]. Animal care and the procedure were approved by the Institutional Animal Care and Use Committee (December $\left.1^{\text {st }}, 2019\right)$. Briefly, mice were euthanized, and both femora and tibiae were aseptically harvested. Bone marrow cells were flushed out with Dulbecco's modified Eagle medium (Gibco, Scotland, UK) supplemented with $10 \%$ heat-inactivated and charcoalstripped fetal bovine serum (Gibco), 1\% penicillin-streptomycin (culture medium). MSCs were identified by flow cytometry and differentiation capability and maintained in the culture medium at $37^{\circ} \mathrm{C}$. Sodium fluoride $(\mathrm{NaF})$ and $17 \beta$ estradiol (ES) served as positive control for different experiments, and PBS was used as vehicle control [22].

2.2. Proliferation Assay. MSCs were seeded in 96-well plates $\left(1 \times 10^{6}\right.$ cells/well $)$ and then incubated for $48 \mathrm{~h}$ with $0.01,0.1$, 1 , and $10 \mu \mathrm{M}$ icariin or icariside II (purity $>97 \%$, previously isolated from Epimedium koreanum Nakai [24]), respectively. Then, 3-[4, 5-dimethylthiazol-2-yl]-2,5 diphenyl tetrazolium bromide (MTT, $5.0 \mathrm{mg} / \mathrm{mL}$, Sigma-Aldrich, St. Louis, MO, USA) was added and further incubated for $4 \mathrm{~h}$. Finally, the medium was replaced with dimethyl sulfoxide. Absorbance was measured at $570 \mathrm{~nm}$.

2.3. Osteogenic Differentiation. Cells were seeded in 48 -well plates $\left(2.5 \times 10^{6}\right.$ cells/well $)$. After reaching $80 \%$ confluence, the medium was changed to the fresh medium with osteogenic supplements (OS: $5 \mathrm{mM} \beta$-glycerophosphate, $0.1 \mu \mathrm{M}$ dexamethasone, and $50 \mathrm{mg} / \mathrm{mL}$ ascorbic acid, SigmaAldrich), ES (10 nM), NaF $(1 \mu \mathrm{M})$, and icariin or icariside II (0.01-10 $\mu \mathrm{M})$. Cultures were maintained for $7 \mathrm{~d}$. Cells were washed with PBS and then lysed by two freeze-thaw cycles. ALP activity and protein content were determined using a commercial ALP kit (Nanjing Jiancheng Bioengineering Institute, Nanjing, China) and by the bicinchoninic acid method (Beyotime Biotechnology, Shanghai, China), respectively, and ALP activity was normalized for total protein concentration [18].

2.4. Alizarin Red S Staining. MSCs were seeded as described in 2.3 and cultured for $21 \mathrm{~d}$ in the OS medium. The presence of mineralized nodules was evaluated by alizarin red S (ARS, Sigma-Aldrich) staining [18]. After being fixed in $70 \%$ ethanol for $30 \mathrm{~min}$, cells were washed with PBS and stained with ARS ( $40 \mathrm{mM}, \mathrm{pH} 4.2$ ) for $30 \mathrm{~min}$. Images were acquired using the Eclipse $\mathrm{Ti}$ microscope (Nikon, Tokyo, Japan) connected to a video camera. The ARS staining was eluted with $10 \%(\mathrm{w} / \mathrm{v})$ cetylpyridium chloride (Sigma-Aldrich) for quantitative analysis. The absorbance was measured at $570 \mathrm{~nm}$.

2.5. Adipogenic Differentiation. Cells were seeded in 48-well plates $\left(3 \times 10^{6}\right.$ cells/well) and cultured for $14 \mathrm{~d}$ in the medium containing adipogenic supplements (AS: $0.1 \mu \mathrm{M}$ dexamethasone and $10 \mathrm{mg} / \mathrm{L}$ insulin, Sigma-Aldrich), ES $(10 \mathrm{nM}), \mathrm{NaF}(1 \mu \mathrm{M})$, and icariin or icariside II $(0.01-10 \mu \mathrm{M})$. Fat droplets were visualized by oil red $\mathrm{O}$ staining [22]. After being fixed with $10 \%$ formalin for $30 \mathrm{~min}$, cells were washed PBS and stained with oil red $\mathrm{O}(0.6 \%(\mathrm{w} / \mathrm{v})$ in $60 \%$ isopropanol) for $15 \mathrm{~min}$. After being eluted with $100 \%(\mathrm{v} / \mathrm{v})$ isopropanol, the absorbance was measured at $510 \mathrm{~nm}$.

2.6. Quantitative Real-Time PCR ( $q P C R$ ) Analysis. Total RNA was obtained from MSCs using TRIzol reagent (Invitrogen, Carlsbad, CA, USA) and reverse transcripted into cDNA using PrimeScript ${ }^{\mathrm{TM}}$ RT reagent kit (TaKaRa, 
Dalian, China). qPCR assay was carried out using SYBR ${ }^{\circledR}$ Premix Ex $\mathrm{Taq}^{\mathrm{TM}}$ II kit (TaKaRa). Primer sequences were according to a previous publication [23]. Relative quantification of mRNA expression was calculated as the fold change using the $2^{-\Delta \Delta C t}$ method [25].

2.7. Enzyme-Linked Immunosorbent Assay (ELISA) Analysis. Levels of phospho-ERK $1 / 2$ and total ERK $1 / 2$ proteins in cell lysis were determined using ERK1/2 (pT202/Y204 + total) ELISA kit (Abcam, Cambridge, UK). Phosphorylated ERK was normalized to total ERK protein and normalized by the control group.

2.8. cDNA Microarray Analysis. MSCs were incubated for $3 \mathrm{~d}$ with icariin $(1 \mu \mathrm{M})$ in the AS or OS medium, respectively. Total RNA was extracted and reverse-transcripted to cDNA with SuperScript III reverse transcriptase (Invitrogen). Cy3dUTP or Cy5-dUTP (Amersham Pharmacia Biotech, Piscataway, NJ) was incorporated during reverse transcription of $40 \mu \mathrm{g}$ of purified total RNA. Different fluorescent-labeled cDNA samples were mixed and competitively hybridized to a homemade cDNA microarray [26], which contained 1000 genes selected from a mouse clone set. Microarrays were scanned using a microarray scanner (ScanArray 4000, GSI Lumonics, MA), and images were analyzed with GenePix Pro 4.0 software (Axon, CA). Genes showing greater than 2fold induction or repression $(\mathrm{Cy} 5 / \mathrm{Cy} 3$ ratios $>2$ or $<0.5)$ were selected for further analysis. The Protein Analysis Through Evolutionary Relationships (PANTHER) classification system was applied to analyze the functional classification and correlated pathways (http://www.pantherdb. org) [27].

2.9. Statistical Analysis. Experiments were performed in three replicates, and all data were expressed as mean\pm standard deviation (SD). The differences between multiple groups were assessed using one-way analysis of variance (ANOVA) or Student's $t$-test. A level of $p<0.05$ was classified as statistically significant.

\section{Results and Discussion}

To evaluate how icariin and icariside II regulate the lineage commitment of MSCs, primary MSCs were isolated, subjected to proliferation assay, and induced to differentiate into adipogenic and osteogenic lineages.

\subsection{Icariin and Icariside II Stimulated the Proliferation of} MSCs. Cells were incubated with icariin, icariside II, $17 \beta-$ estradiol (ES), and $\mathrm{NaF}$ for $2 \mathrm{~d}$. As shown in Figure 1, $10 \mathrm{nM}$ ES increased the proliferation rate of MSCs to $15.2 \%$ versus PBS control $(p<0.05)$. In contrast, $0.01-10 \mu \mathrm{M}$ icariin and icariside II more potently stimulated the proliferation of MSCs up to $30 \%(p<0.05)$, respectively. The maximum effect was obtained at $0.1 \mu \mathrm{M}$ for both icariin and icariside II, showing a tendency to decrease with increasing concentrations. Wei et al. [28] reported that icariin $(0.1 \mu \mathrm{M})$ effectively induced the proliferation of rat MSCs from 1 to 14 days $(p<0.05)$. Studies also indicated that increasing icariin concentrations, for instance, over $20 \mu \mathrm{M}$ may exhibit a negative effect on rat MSCs cell growth [29], and $0.001 \mu \mathrm{M}$ had no significant effect [30]. Therefore, the optimal concentrations for cell proliferation may be $0.01-10 \mu \mathrm{M}$.

\subsection{Icariin and Icariside II Promoted the Osteogenic Differ-} entiation of MSCs. Previously, our group [22] and Zhang et al. [10] found that total flavonoids of Herba Epimedii significantly stimulated the osteogenic differentiation of mouse and human MSCs, respectively, among which icariin and icariside II were the main constituents. Another study indicated that $1 \mu \mathrm{M}$ icariin may induce osteogenesis of rat MSCs by increasing ALP activity and calcium deposition [30]; however, no other concentrations and positive control were evaluated. Osteogenesis of MSCs was first quantified by monitoring ALP activity, an early marker. As shown in Figure 2, both ES and $\mathrm{NaF}$ potently increased ALP activity to 3.1 and 2.2-fold, respectively $(p<0.01)$. Icariin $(0.1-10 \mu \mathrm{M})$ stimulated ALP of MSCs in a concentration-dependent manner. Icariside II $(0.01-10 \mu \mathrm{M})$ also significantly induced the ALP activity of MSCs, among which $0.1 \mu \mathrm{M}$ was the most potent.

Osteogenic differentiation of MSCs was further characterized by ARS staining to detect extracellular matrix (ECM) mineralization (Figure 3). Osteogenic supplements intensively increased calcium deposition as indicated by ARS staining (Figure 3(a)). In addition, a robust number of mineralized nodules were found in $\mathrm{NaF}$, icariin, and icariside II groups (Figures 3(b)-3(d)).

Quantification of ARS staining confirmed that $1 \mu \mathrm{M} \mathrm{NaF}$ potently increased the calcium deposition in ECM during osteogenic differentiation of MSCs (Figure 3(e)). Similarly, $0.1-10 \mu \mathrm{M}$ icariin significantly promoted the calcium deposition in ECM (Figure 3(e), $p<0.05$ ), which was confirmed by another study that $0.01-1 \mu \mathrm{M}$ icariin stimulated the calcium deposition in rat MSCs [28]. In addition, calcium deposition in ECM was also significantly induced by $0.01-10 \mu \mathrm{M}$ icariside II in a concentration-dependent manner (Figure 3(f)). At the concentration of $10 \mu \mathrm{M}$, both icariin and icariside II reached the maximum effect, increasing the calcium content by $37.9 \%$ and $30.2 \%$, respectively $(p<0.01)$. Taken together, both $0.1-10 \mu \mathrm{M}$ icariin and $0.01-10 \mu \mathrm{M}$ icariside II may significantly promote osteogenesis of MSCs by enhancing early marker-ALP activity and final marker-ECM mineralization.

3.3. Icariin and Icariside II Suppressed the Adipogenic Differentiation of MSCs. Although Herba Epimedii has long been used in traditional Chinese medicine for the bone and joints, recent data have shown that Herba Epimedii may offer potential benefits for obesity. Herba Epimedii extract $(100,200 \mu \mathrm{g} / \mathrm{mL})$ and icariin $(50,100 \mu \mathrm{M})$ were reported to inhibit the adipocyte differentiation of 3T3-L1 preadipocytes by decreasing the expression of the adipogenic transcription factors [31]. One study found that icariin was a novel peroxisome proliferator-activated receptor-alpha (PPARa) agonist which activated lipid metabolism gene expressions 


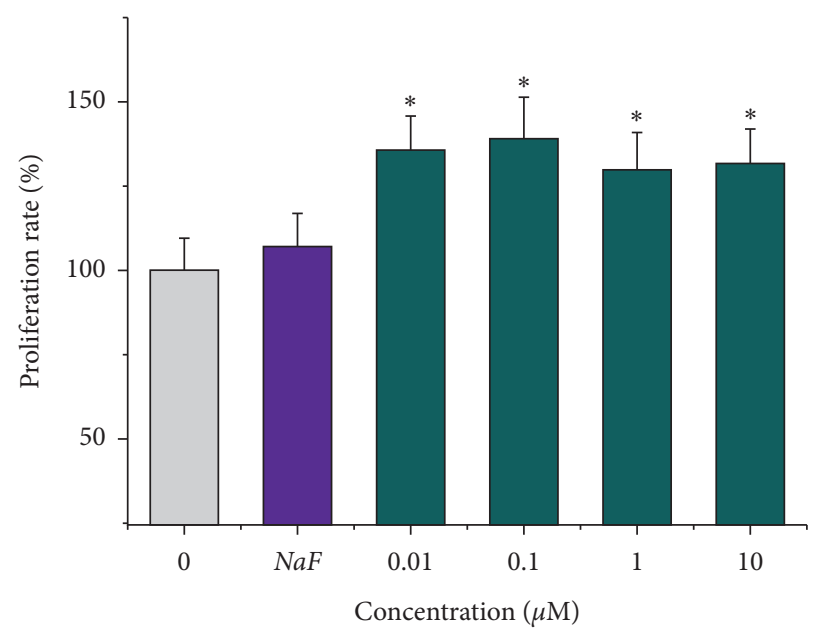

(a)

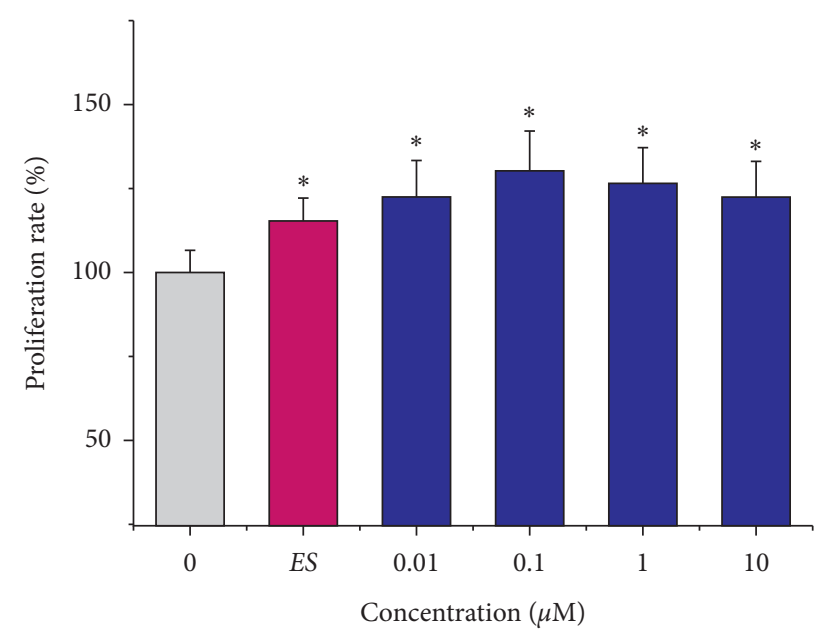

(b)

FIGURE 1: Icariin (a) and icariside II (b) stimulated the proliferation of MSCs. NaF, $1 \mu \mathrm{M}$; ES, $10 \mathrm{nM} 17 \beta$-estradiol. ${ }^{*} \mathrm{P}<0.05$ vs. control.

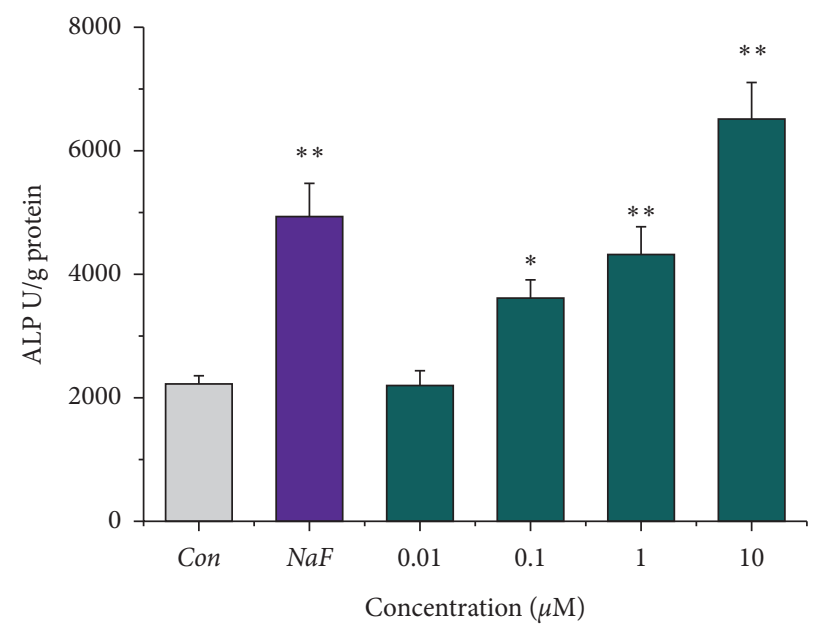

(a)

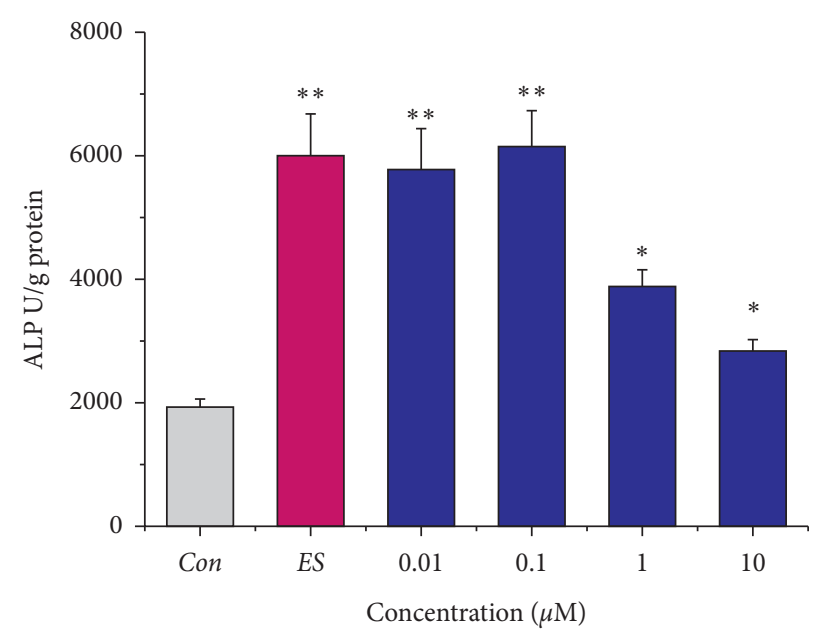

(b)

FIGURE 2: Icariin (a) and icariside II (b) increased ALP activity during osteogenesis of MSCs. NaF, $1 \mu \mathrm{M}$; ES, $10 \mathrm{nM} 17 \beta$-estradiol. ${ }^{*} P<0.05$ and ${ }^{* *} p<0.01$ vs. PBS control.

in mice [32]. Another study reported that icariin induced irisin/FNDC5 expression in $\mathrm{C} 2 \mathrm{C} 12$ cells, indicating that icariin may protect against obesity [33]. Previously, our group found that total flavonoids of Herba Epimedii significantly suppressed the adipogenic differentiation of mouse MSCs [22], and icariin may suppress adipocytic transdifferentiation of primary osteoblasts [18]. Therefore, available data suggest that icariin may play an essential role in adipogenesis.

MSCs were capable of adipogenic differentiation in the adipogenic supplements (AS) medium characterized by oil red $\mathrm{O}$ staining. In Figures $4(\mathrm{a})-4(\mathrm{~d})$, plenty of reddishbrown droplets were formed and accumulated in the AS group, which were decreased by the addition of test samples. Quantitative analysis revealed that $0.1-10 \mu \mathrm{M}$ icariin and $1-10 \mu \mathrm{M}$ icariside II significantly suppressed the adipogenic differentiation of MSCs (Figures 4(e)-4(f), $p<0.05$ ), which was slightly increased with concentration. Notably, the adipogenic inhibitory effect of experimental groups was as follows: icariin $(10 \mu \mathrm{M})>$ icariside II $(10 \mu \mathrm{M})>\operatorname{ES}(10 \mathrm{nM})$.

3.4. Gene Expression. The differentiation balance of MSCs, especially to osteogenic and adipogenic lineages, is crucial to bone hematopoiesis. Runt-related transcription factor 2 (Runx2) and Pparg are generally regarded as the key regulators of osteogenesis and adipogenesis, respectively. Studies have demonstrated an inverse relationship between Runx2 and Pparg expression during osteogenesis and adipogenesis of MSCs [19, 34]. Our previous studies showed that flavonoids of Herba Epimedii promoted the osteogenesis of MSCs by increasing the Runx2 and bone 

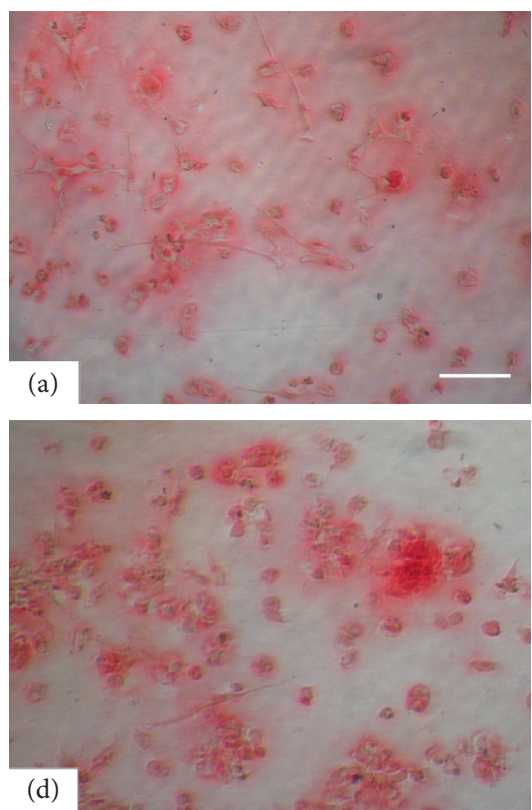
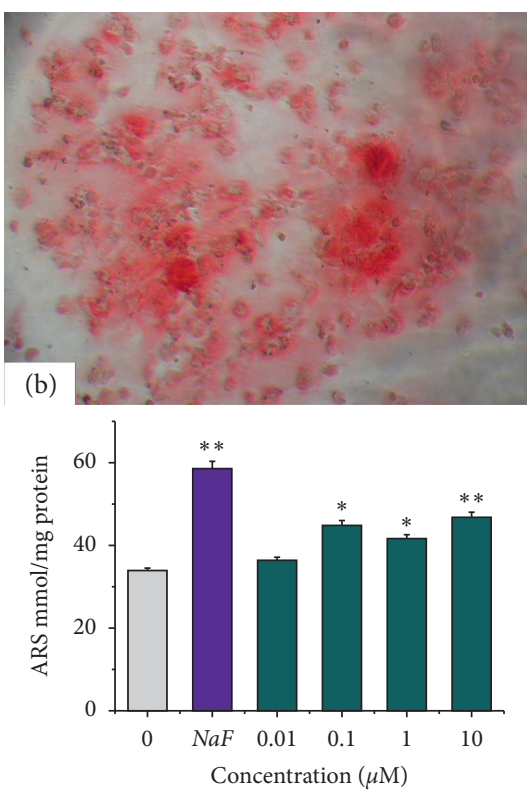

(e)
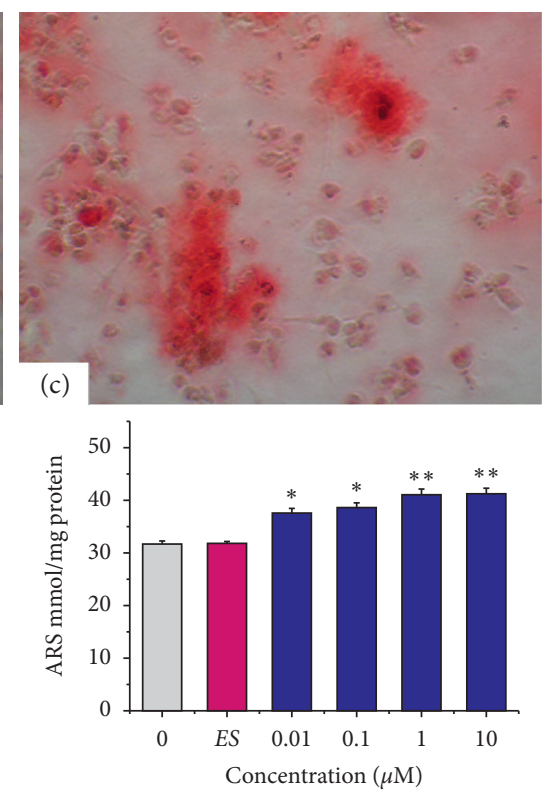

(f)

FIGURE 3: Quantification of alizarin red S staining during osteogenesis of MSCs. (a-d). Alizarin red S staining (100x): (a) Osteogenic supplements (OS) medium. (b) OS + NaF $(1 \mu \mathrm{M})$. (c) OS + icariin $(1 \mu \mathrm{M})$. (d) OS + icariside II $(1 \mu \mathrm{M})$. (e-f) Quantification of ARS staining: (e) Icariin. (f) Icariside II. ${ }^{*} P<0.05$ and ${ }^{* *} p<0.01$ vs. the OS group. Scale bar $=20 \mu \mathrm{M}$.
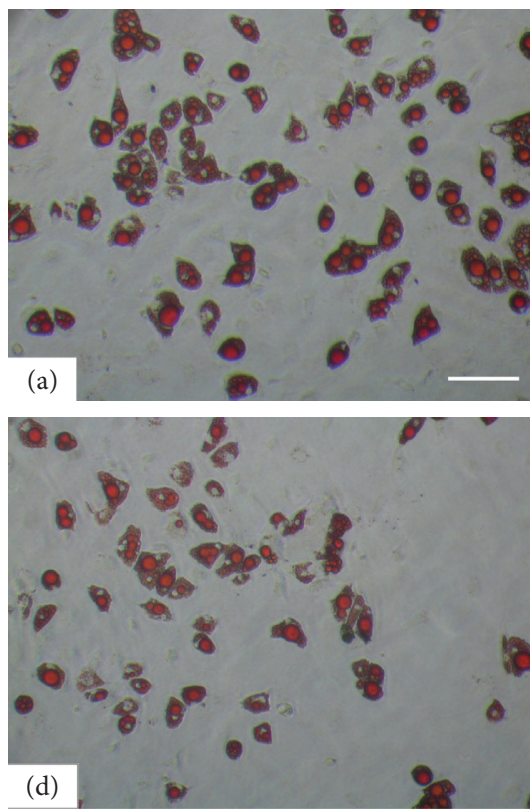
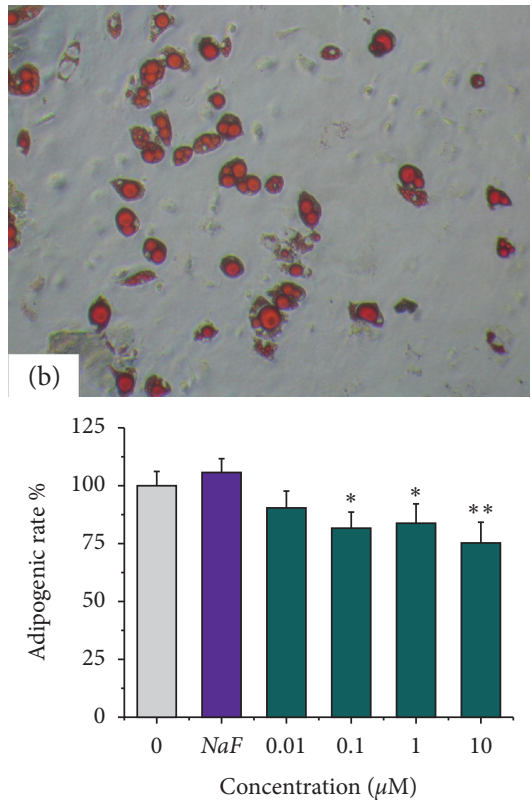

(e)
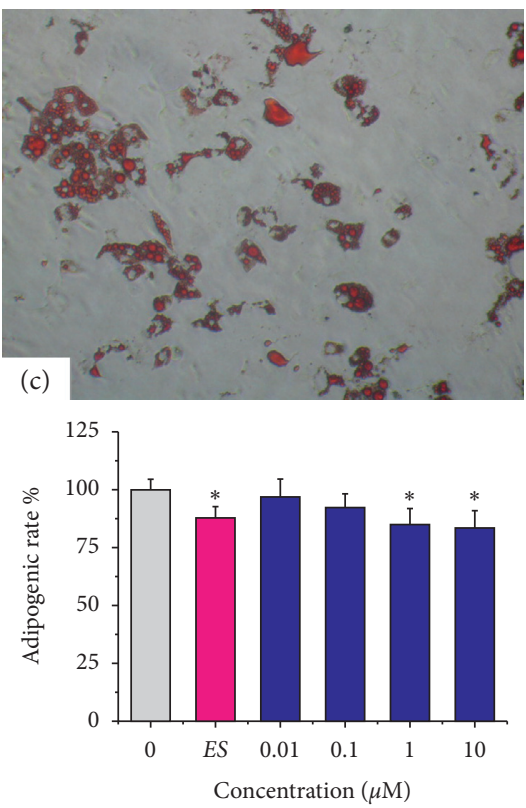

(f)

FIGURE 4: Adipocyte-like cell formation during adipogenesis of MSCs. (a-d) Oil red O staining (100×). (a) AS medium. (b) AS + ES (10 nM). (c) AS + icariin $(1 \mu \mathrm{M})$. (d) AS + icariside II $(1 \mu \mathrm{M})$. (e-f) Quantification of oil red O staining: (e) Icariin. (f) Icariside II. ${ }^{*} P<0.05$ and ** $p<0.01$ vs. the AS group. Scale bar $=40 \mu \mathrm{M}$.

morphogenetic protein $2(B m p 2)$ gene expression while downregulating the expression of Pparg [22].

As shown in Figure 5, qPCR assay indicated that icariin and icariside II induced the osteogenic and adipogenic bidirectional differentiation of MSCs through modulating osteogenic and adipogenic-related gene expressions. The addition of ES, icariin, and icariside II significantly upregulated the expression of Runx2, collagen type 1 (Col1), and Bmp2 (Figure 5(a)). It is noticeable that $10 \mu \mathrm{M}$ icariin upregulated Runx2, Col1, and Bmp2 expression to 2.1, 2.5, and 4.1-fold, respectively. No significant difference between icariin and icariside II was found. 

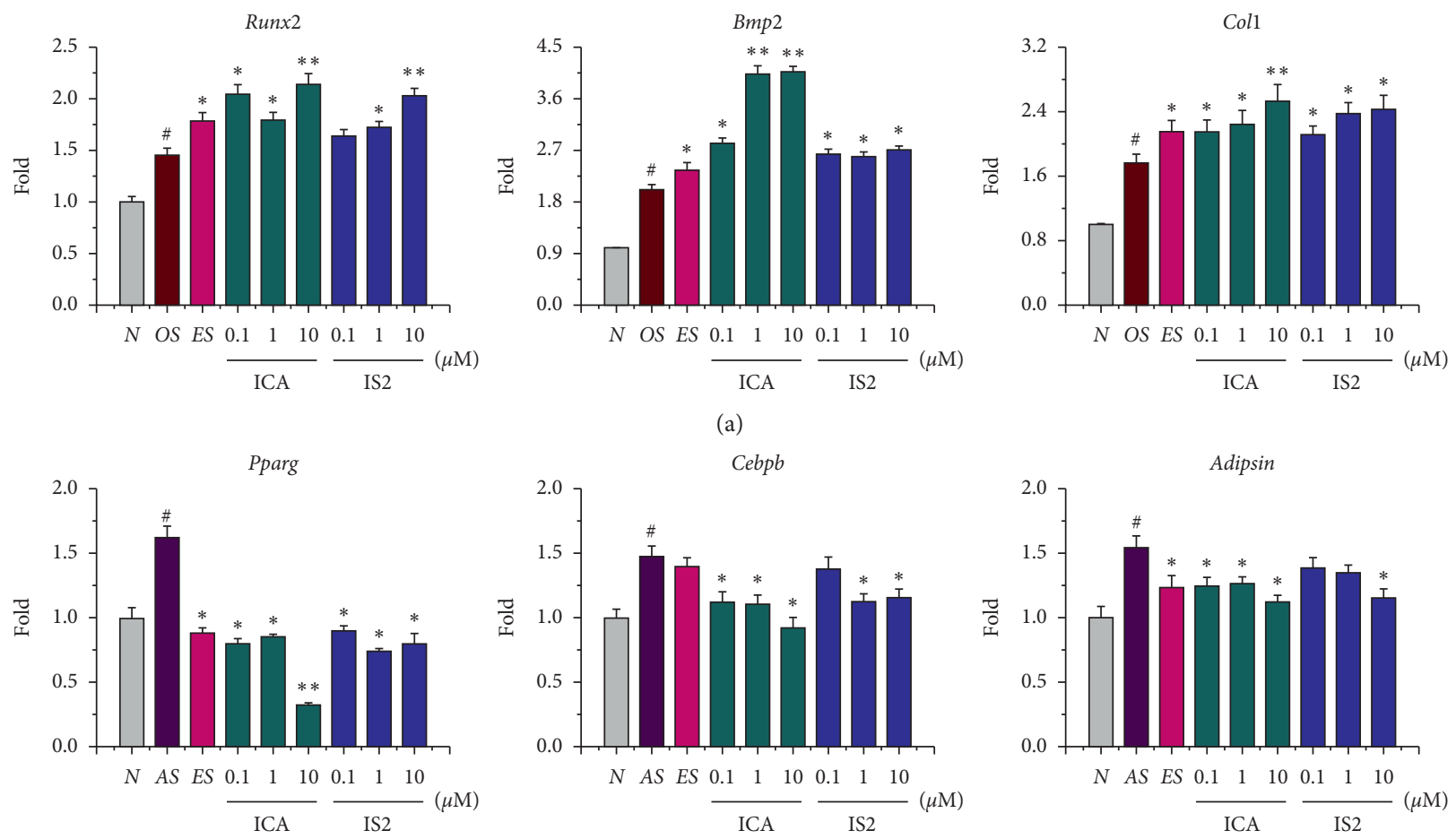

(b)

FIGURE 5: Representative gene expression during osteogenesis (a) and adipogenesis (b) of MSCs in the presence of icariin and icariside II. AS, adipogenic supplement; OS, osteogenic supplement; ES, $10 \mathrm{nM} 17 \beta$-estradiol; N, growth medium; ICA, icariin; IS2, icariside II. ${ }^{\#} P<0.01$ vs. the $\mathrm{N}$ group. ${ }^{*} P<0.05$ and ${ }^{* *} p<0.01$ vs. the AS/OS group.

Similarly, representative adipogenic marker genes, Pparg, CCAAT/enhancer-binding protein beta $(\mathrm{Ce} b p b)$, and Adipsin, were markedly upregulated in the AS medium (Figure 5(b)). In comparison, ES treatment decreased the expression of Pparg and Adipsin. Both icariin and icariside II downregulated the expression of Pparg, Cebpb, and Adipsin. For instance, icariin $(10 \mu \mathrm{M})$ and icariside II $(1 \mu \mathrm{M})$ decreased the expression of Pparg by $67.9 \%$ and $26.2 \%$ versus the control group, respectively.

\subsection{ERK Signaling Was Involved during MSCs Osteogenic and} Adipogenic Differentiation. The ERK signaling pathway is critically involved in the commitment of MSCs into the osteogenic lineage [35]. ERK signaling could enhance ALP activity in osteoblast progenitor cells and MSCs by activating the osteogenic-related transcription regulators [36, 37]. Jaiswal et al. [21] found that inactivation of ERK blocked the osteogenic differentiation but induced the adipogenesis of adult human MSCs.

In Figures 6(a)-6(c), pretreatment with $10 \mu \mathrm{M}$ U0126, specific ERK1/2 inhibitor, decreased the above biological effects of icariin and icariside II on ALP activity and gene expression of Runx2 and Pparg $(P<0.05)$. In addition, icariin and icariside II treatment activated ERK signaling by increasing the phosphorylation of ERK (Figure 6(d), $P<0.01$ ), which suggests that icariin and icariside II may drive the osteogenesis and adipogenesis of MSCs through ERK signaling.

3.6. Gene Expression Profiling during MSCs Osteogenic and Adipogenic Differentiation. According to PANTHER analysis, 27 differentially expressed genes during MSCs osteogenesis were categorized into 41 biological processes and 24 molecular functions (Figure 7, Supplementary Materials Table 1). More specifically, important biological processes were the cellular process $(26.8 \%)$, biological regulation (19.5\%), metabolic process (17.1\%), and response to stimulus (12.2\%). In addition, binding accounted for as much as 50\% of molecular functions. Similarly, 30 differentially regulated genes during MSCs adipogenesis were involved in 40 biological processes and 30 molecular functions (Figure 8, Supplementary Materials Table 2). Most of the above genes were related to the cellular process (32.5\%), metabolic process $(27.5 \%)$, and biological regulation $(20.0 \%)$ in biological process and binding (36.7\%) and catalytic activity $(33.3 \%)$ in molecular function (Figure 8). The effects of icariin and icariside II on the above genes were under investigation. For example, during osteogenic differentiation of MSCs, icariin upregulated Srebf1 and Psma6 to 2.20 and 3.07-fold, respectively, while during adipogenic differentiation of MSCs, icariin downregulated Marcks and Lpl to 0.49 and 0.36 -fold, respectively. 


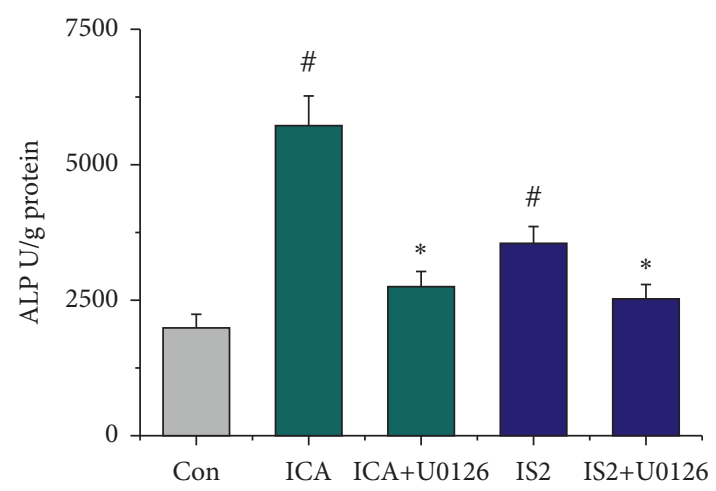

(a)

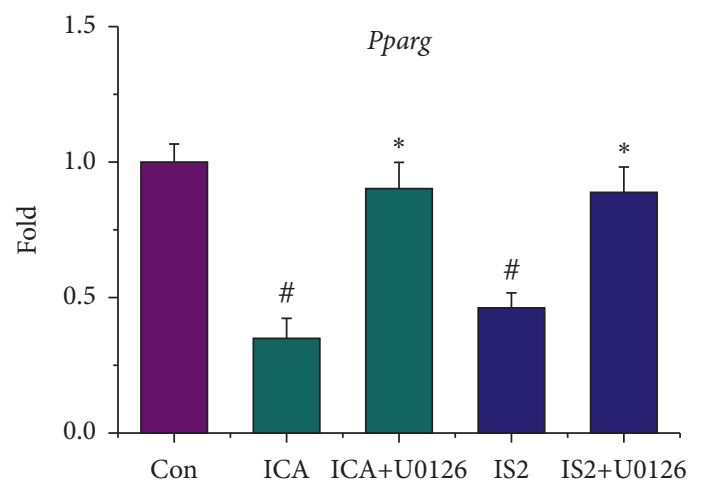

(c)

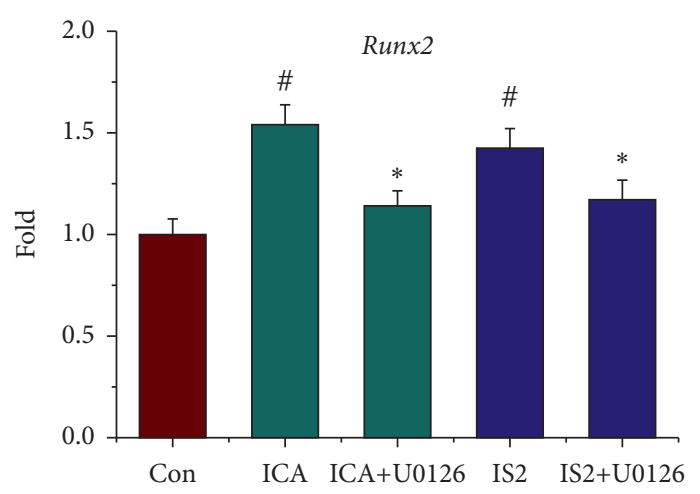

(b)

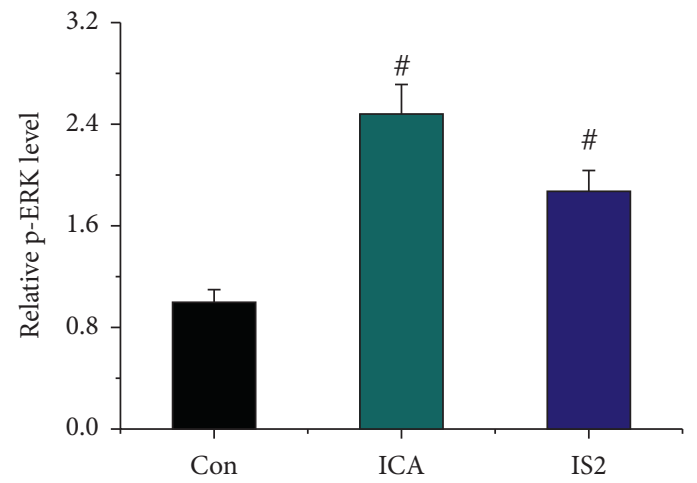

(d)

FIGURE 6: ERK signaling was activated during osteogenesis and adipogenesis of MSCs in the presence of icariin and icariside II. (a) ALP activity. (b-c) Gene expression of Runx2 and Pparg. (d) Level of ERK phosphorylation by ELISA. ICA, icariin; IS2, icariside II; U0126, ERK1/2 inhibitor. ${ }^{\#} P<0.01$ vs. the control group. ${ }^{*} P<0.05$ vs. the ICA/IS2 group.
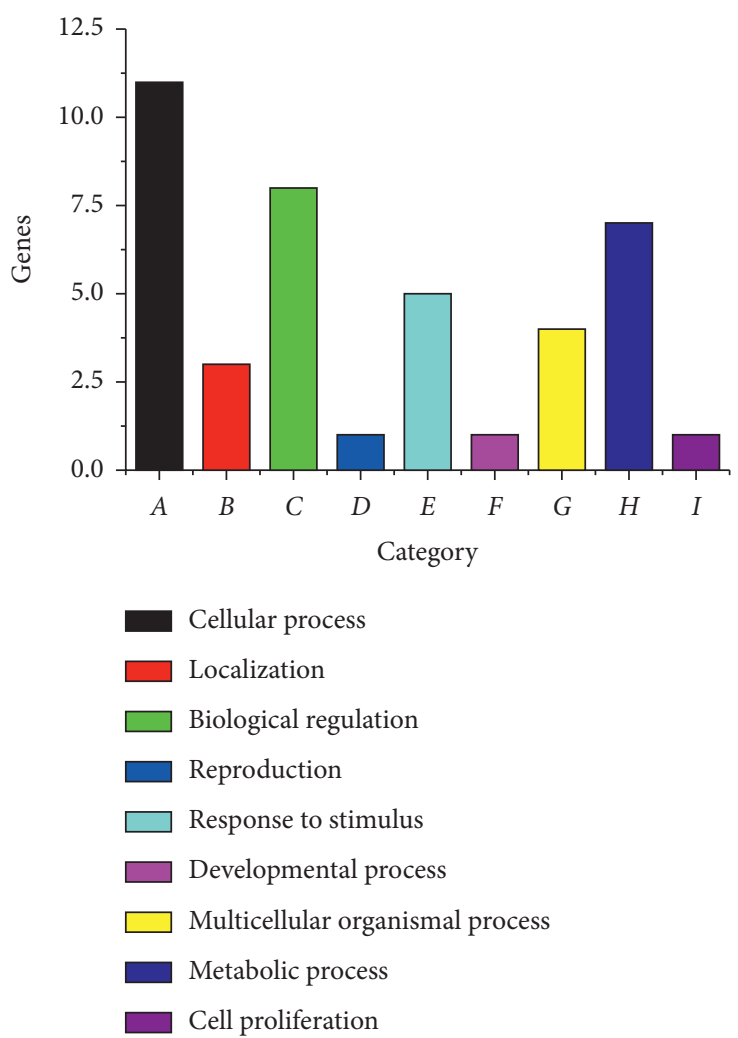

(a)
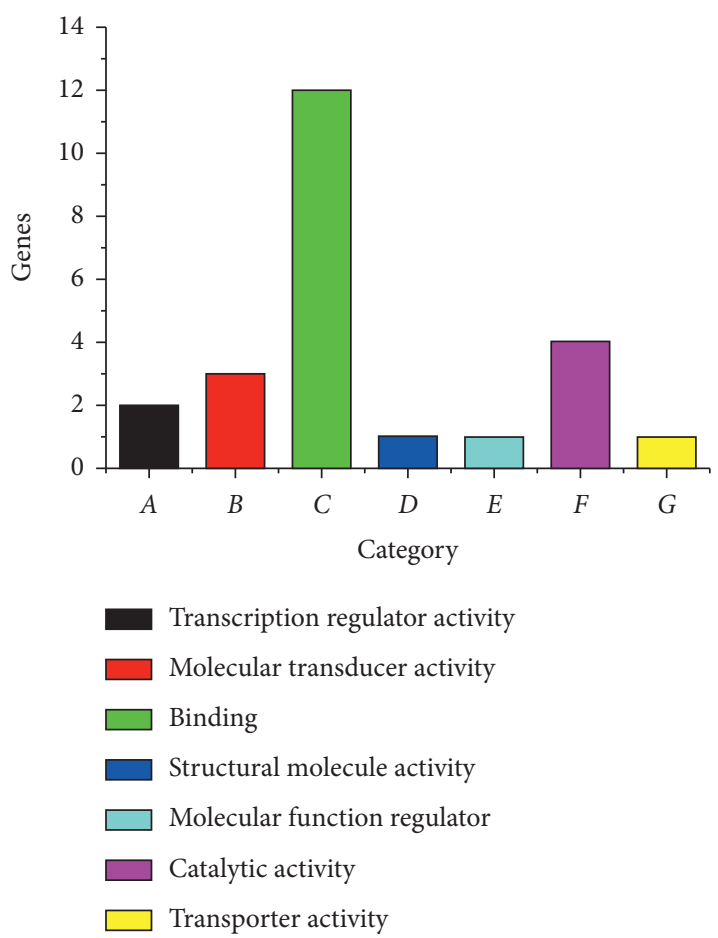

Figure 7: PANTHER functional analysis during osteogenic differentiation of MSCs. (a) Biological process. (b) Molecular function. 


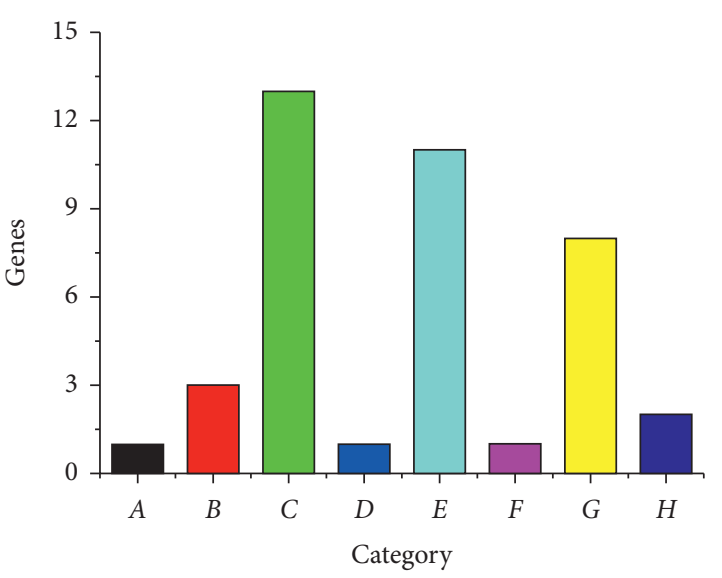

Reproduction
$\square$ Response to stimulus
$\square$ Cellular process
$\square$ Multicellular organismal process
$\square$ Metabolic process
$\square$ Cell proliferation
$\square$ Biological regulation
$\square$ Localization

(a)
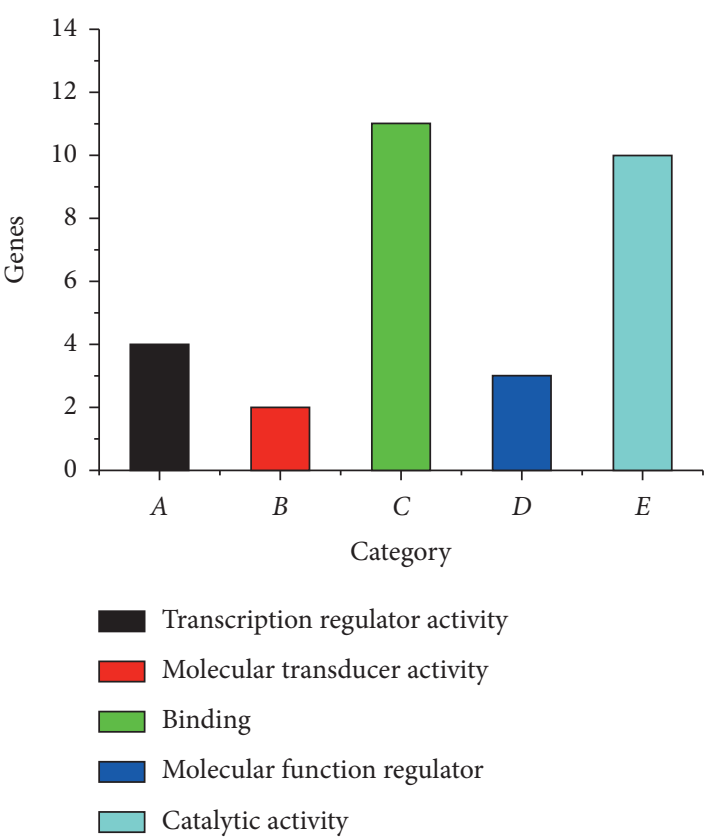

(b)

FIGURE 8: PANTHER functional analysis during adipogenic differentiation of MSCs. (a) Biological process. (b) Molecular function.

\section{Conclusion}

MSCs are self-renewing, multipotent precursors to various cell lineages, which have shown great promise in the field of tissue engineering and regenerative medicine. An imbalance between osteogenesis and adipogenesis of MSCs may lead to various metabolic diseases. Our results revealed the reciprocal regulation of osteogenesis and adipogenesis of MSCs by icariin and its main metabolite icariside II through ERK signaling. The gene expression profiles indicated that 57 genes were involved in the lineage commitment of MSCs. Previous studies strongly showed that Herba Epimedii flavonoids might restore bone homeostasis by promoting bone formation and suppressing bone resorption. These findings, taken together, contribute to a comprehensive evaluation of the osteoprotective effects of Herba Epimedii flavonoids. In summary, Herba Epimedii, especially its flavonoid components, has broad prospects of application in osteoporosis prevention and healthcare.

\section{Data Availability}

The data used to support the findings of this study are available from the corresponding author upon request.

\section{Conflicts of Interest}

The authors declare that they have no conflicts of interest.

\section{Authors' Contributions}

D. Zhang and $\mathrm{H}$. Wang designed the study and drafted the manuscript. N. Zhao, J. Lin, and J. Du collected the data. C. Wan and J. Du analyzed the results. All authors read and approved the final version for submission.

\section{Acknowledgments}

This work was financially supported by the National Natural Science Foundation of China (81402933), Natural Science Foundation of Guangdong Province, China (2021A1515011120), Research Foundation of Administration of Traditional Chinese Medicine of Guangdong Province, China (20192042 and 20201181), Medical Scientific Research Foundation of Guangdong Province, China (B2019047), Research Foundation of Guangdong Medical University (GDMUZ201805), The Public Service Platform of South China Sea for R\&D Marine Biomedicine Resources from Guangdong Medical University (2HC18018), Sanming Project of Medicine in Shenzhen (SZZYSM202106003), High-Level Professional in Shenzhen, Yangfan Plan for High-Level Talents of Guangdong Province, and High-Level Talents in Shenzhen Baoan.

\section{Supplementary Materials}

Figure 1. Chemical structure of icariin and icariside II. Table 1. Gene expression profiles during osteogenic differentiation of MSCs by cDNA microarray. Table 2. Gene expression profiles during adipogenic differentiation of 
MSCs by cDNA microarray. Supplementary material related to this article can be found in the online version. (Supplementary Materials)

\section{References}

[1] M. R. Allen and D. B. Burr, "Bone modeling and remodeling," in Basic and Applied Bone Biology, M. R. Allen and D. B. Burr, Eds., Academic Press, Cambridge, MA, USA, pp. 75-90, 2013.

[2] R. M. Arceo-Mendoza and P. M. Camacho, "Postmenopausal osteoporosis," Endocrinology and Metabolism Clinics of North America, vol. 50, no. 2, pp. 167-178, 2021.

[3] W. Słupski, P. Jawień, and B. Nowak, "Botanicals in postmenopausal osteoporosis," Nutrients, vol. 13, no. 5, p. 1609, 2021.

[4] L. Wang, Y. Li, Y. Guo et al., "Herba epimedii: an ancient Chinese herbal medicine in the prevention and treatment of osteoporosis," Current Pharmaceutical Design, vol. 22, no. 3, pp. 328-349, 2016.

[5] F. Xie, C.-F. Wu, W.-P. Lai et al., "The osteoprotective effect of Herba epimedii (HEP) extract in vivo and in vitro," Evidencebased Complementary and Alternative Medicine, vol. 2, no. 3, pp. 353-361, 2005.

[6] W.-F. Chen, S.-K. Mok, X.-L. Wang et al., "Total flavonoid fraction of the Herba epimedii extract suppresses urinary calcium excretion and improves bone properties in ovariectomised mice," British Journal of Nutrition, vol. 105, no. 2, pp. 180-189, 2011.

[7] H. Nian, M.-H. Ma, S.-S. Nian, and L.-L. Xu, "Antiosteoporotic activity of icariin in ovariectomized rats," Phytomedicine, vol. 16, no. 4, pp. 320-326, 2009.

[8] G. Chen, C. Wang, J. Wang et al., "Antiosteoporotic effect of icariin in ovariectomized rats is mediated via the Wnt/ $\beta$-catenin pathway," Experimental and Therapeutic Medicine, vol. 12, no. 1, pp. 279-287, 2016.

[9] H.-H. Xiao, C.-Y. Fung, S.-K. Mok et al., "Flavonoids from Herba epimedii selectively activate estrogen receptor alpha $(\mathrm{ER} \alpha)$ and stimulate ER-dependent osteoblastic functions in UMR-106 cells," The Journal of Steroid Biochemistry and Molecular Biology, vol. 143, pp. 141-151, 2014.

[10] J. F. Zhang, G. Li, C. L. Meng et al., “Total flavonoids of Herba epimedii improves osteogenesis and inhibits osteoclastogenesis of human mesenchymal stem cells," Phytomedicine, vol. 16, no. 6-7, pp. 521-529, 2009.

[11] D. Zhang, J. Zhang, C. Fong, X. Yao, and M. Yang, "Herba epimedii flavonoids suppress osteoclastic differentiation and bone resorption by inducing G2/M arrest and apoptosis," Biochimie, vol. 94, no. 12, pp. 2514-2522, 2012.

[12] J.-F. Zhang, G. Li, C.-Y. Chan et al., "Flavonoids of Herba Epimedii regulate osteogenesis of human mesenchymal stem cells through BMP and Wnt/ $\beta$-catenin signaling pathway," Molecular and Cellular Endocrinology, vol. 314, no. 1, pp. 70-74, 2010.

[13] D. Liu, Y. Ye, L. Xu, W. Yuan, and Q. Zhang, "Icariin and mesenchymal stem cells synergistically promote angiogenesis and neurogenesis after cerebral ischemia via PI3K and ERK1/ 2 pathways," Biomedicine \& Pharmacotherapy, vol. 108, pp. 663-669, 2018.

[14] X. Yao, X. Jing, J. Guo et al., "Icariin protects bone marrow mesenchymal stem cells against iron overload induced dysfunction through mitochondrial fusion and fission, PI3K/Akt/ mTOR and MAPK pathways," Frontiers in Pharmacology, vol. 10, p. 163, 2019.
[15] Z. Luo, M. Liu, L. Sun, and F. Rui, "Icariin recovers the osteogenic differentiation and bone formation of bone marrow stromal cells from a rat model of estrogen deficiencyinduced osteoporosis," Molecular Medicine Reports, vol. 12, no. 1, pp. 382-388, 2015.

[16] S. Xu, J. Yu, J. Zhan, L. Yang, L. Guo, and Y. Xu, "Pharmacokinetics, tissue distribution, and metabolism study of icariin in rat," BioMed Research International, vol. 2017, Article ID 4684962, 17 pages, 2017.

[17] M. Wang, H. Gao, W. Li, and B. Wu, "Icariin and its metabolites regulate lipid metabolism: from effects to molecular mechanisms," Biomedicine \& Pharmacotherapy, vol. 131, Article ID 110675, 2020.

[18] D. Zhang, C. Fong, Z. Jia, L. Cui, X. Yao, and M. Yang, "Icariin stimulates differentiation and suppresses adipocytic transdifferentiation of primary osteoblasts through estrogen receptor-mediated pathway," Calcified Tissue International, vol. 99, no. 2, pp. 187-198, 2016.

[19] Y. K. Luu, E. Capilla, C. J. Rosen et al., "Mechanical stimulation of mesenchymal stem cell proliferation and differentiation promotes osteogenesis while preventing dietary-induced obesity," Journal of Bone and Mineral Research, vol. 24, no. 1, pp. 50-61, 2009.

[20] X. Yang, X. Cai, J. Wang et al., "Mechanical stretch inhibits adipogenesis and stimulates osteogenesis of adipose stem cells," Cell Proliferation, vol. 45, no. 2, pp. 158-166, 2012.

[21] R. K. Jaiswal, N. Jaiswal, S. P. Bruder, G. Mbalaviele, D. R. Marshak, and M. F. Pittenger, "Adult human mesenchymal stem cell differentiation to the osteogenic or adipogenic lineage is regulated by mitogen-activated protein kinase," Journal of Biological Chemistry, vol. 275, no. 13, pp. 9645-9652, 2000.

[22] D. Zhang, L. Liu, Z. Jia, X. Yao, and M. Yang, "Flavonoids of Herba epimedii stimulate osteogenic differentiation and suppress adipogenic differentiation of primary mesenchymal stem cells via estrogen receptor pathway," Pharmaceutical Biology, vol. 54, no. 6, pp. 954-963, 2016.

[23] D. Liu, C. Yi, C.-C. Fong et al., "Activation of multiple signaling pathways during the differentiation of mesenchymal stem cells cultured in a silicon nanowire microenvironment," Nanomedicine: Nanotechnology, Biology and Medicine, vol. 10, no. 6, pp. 1153-1163, 2014.

[24] D. W. Zhang, Y. Cheng, N. L. Wang, J. C. Zhang, M. S. Yang, and X. S. Yao, "Effects of total flavonoids and flavonol glycosides from Epimedium koreanum Nakai on the proliferation and differentiation of primary osteoblasts," Phytomedicine, vol. 15, no. 1-2, pp. 55-61, 2008.

[25] K. J. Livak and T. D. Schmittgen, "Analysis of relative gene expression data using real-time quantitative PCR and the $2^{-\Delta \Delta \mathrm{CT}}$ method," Methods, vol. 25, no. 4, pp. 402-408, 2001.

[26] C. C. Fong, Q. Zhang, Y. F. Shi, R. S. Wu, W. F. Fong, and M. Yang, "Effect of hypoxia on RAW264.7 macrophages apoptosis and signaling," Toxicology, vol. 235, no. 1-2, pp. 52-61, 2007.

[27] H. Mi, A. Muruganujan, J. T. Casagrande, and P. D. Thomas, "Large-scale gene function analysis with the PANTHER classification system," Nature Protocols, vol. 8, no. 8, pp. 1551-1566, 2013.

[28] Q. Wei, M. He, M. Chen et al., "Icariin stimulates osteogenic differentiation of rat bone marrow stromal stem cells by increasing TAZ expression," Biomedicine \& Pharmacotherapy, vol. 91, pp. 581-589, 2017. 
[29] Y. Wu, L. Xia, Y. Zhou, Y. Xu, and X. Jiang, "Icariin induces osteogenic differentiation of bone mesenchymal stem cells in a MAPK-dependent manner," Cell Proliferation, vol. 48, no. 3, pp. 375-384, 2015.

[30] J. M. Huang, Y. Bao, W. Xiang et al., "Icariin regulates the bidirectional differentiation of bone marrow mesenchymal stem cells through canonical wnt signaling pathway," Evidence-Based Complementary and Alternative Medicine: eCAM, vol. 2017, Article ID 8085325, 12 pages, 2017.

[31] Y.-Y. Han, M.-Y. Song, M.-S. Hwang, J.-H. Hwang, Y.-K. Park, and H.-W. Jung, "Epimedium koreanum Nakai and its main constituent icariin suppress lipid accumulation during adipocyte differentiation of 3T3-L1 preadipocytes," Chinese Journal of Natural Medicines, vol. 14, no. 9, pp. 671-676, 2016.

[32] Y.-F. Lu, Y.-Y. Xu, F. Jin, Q. Wu, J.-S. Shi, and J. Liu, "Icariin is A PPAR $\alpha$ activator inducing lipid metabolic gene expression in mice," Molecules, vol. 19, no. 11, pp. 18179-18191, 2014.

[33] S.-Q. Chen, L.-N. Ding, N.-X. Zeng et al., "Icariin induces irisin/FNDC5 expression in $\mathrm{C} 2 \mathrm{C} 12$ cells via the AMPK pathway," Biomedicine \& Pharmacotherapy, vol. 115, Article ID 108930, 2019.

[34] Y. F. Lin, W. Jing, L. Wu et al., "Identification of osteo-adipo progenitor cells in fat tissue," Cell Proliferation, vol. 41, no. 5, pp. 803-812, 2008.

[35] C. Jin, T. Shuai, and Z. Tang, "HSPB7 regulates osteogenic differentiation of human adipose derived stem cells via ERK signaling pathway," Stem Cell Research \& Therapy, vol. 11, no. 1, p. $450,2020$.

[36] C. Ge, G. Xiao, D. Jiang, and R. T. Franceschi, "Critical role of the extracellular signal-regulated kinase-MAPK pathway in osteoblast differentiation and skeletal development," Journal of Cell Biology, vol. 176, no. 5, pp. 709-718, 2007.

[37] J. Lou, Y. Tu, S. Li, and P. R. Manske, "Involvement of ERK in BMP-2 induced osteoblastic differentiation of mesenchymal progenitor cell line C3H10T1/2," Biochemical and Biophysical Research Communications, vol. 268, no. 3, pp. 757-762, 2000. 